ROCZNIKI HUMANISTYCZNE

Tom LXIX, zeszyt 5 - 2021

DOI: http://doi.org/10.18290/rh21695-9

JOACHIM GRZEGA

\title{
EUROLINGUISTISCHER BLICK AUF WILLY BRANDT - FRIEDEN FÖRDERN DURCH ÜBERWINDUNG RHETORISCHER GRENZEN
}

\begin{abstract}
A b stract. 1969-1971 sind wichtige Jahre in der Beendigung des Kalten Krieges: Beginn und erste Früchte der neuen Auslandspolitik Willy Brandts. Anhand von Regierungserklärungen, Vertragstexten, Gesprächsprotokollen und Fernsehansprachen wird exemplarisch gezeigt, dass ein Baustein für den Erfolg Brandts in der Überwindung bisheriger rhetorischer Grenzen liegt. Seine Rhetorik zeichnet sich aus durch: (1) zeitnahes Umsetzen von Worten in Taten, (2) Bewusstsein um kulturspezifische Symbolwörter, (3) Schaffen neuer positiver Symbolwörter, (4) adressatenbewusste Wort-Variation, (5) (gute) Kommunikation zu Machtinhabern.
\end{abstract}

Schlüsselwörter: Willy Brandt; Frieden fördern; Grenzen der Rhetorik; Eurolinguistik.

\section{EINLEITUNG}

1969 Beginn von Willy Brandts „Politik der kleinen Schritte“ gegenüber dem Osten (genannt Neue Ostpolitik), 1970 Unterzeichnung von Moskauer und Warschauer Vertrag, 1971 Verleihung des Friedensnobelpreises an Brandt - diese Ereignisse verzeichnen den Anfang vom Ende des Kalten Kriegs zwischen West und Ost. Sie jähren sich in diesen Jahren zum 50. Mal und werden doch oft überlagert von den Feierlichkeiten zu den Ereignissen von 1989 bis 1991. Ein Teil des Erfolgs von Brandts Neuer Ostpolitik, die er selbst lieber „Politik der aktiven Friedenssicherung“ genannt hat (s. Fischer, Willy Brandt 53), ist verbunden mit neuen rhetorischen beziehungsweise kommunikativen Strategien. Dies soll hier anhand von qualitativen Analysen

Prof. Dr. phil. JoAchim Grzega - Universität Eichstätt, Sprach- und Literaturwissenschaftliche Fakultät; Korrespondenzadresse: Universitätsallee 1, D-85072 Eichstätt / Volkshochschule Donauwörth; Korrespondenzadresse: Spindeltal 5, D-86609 Donauwörth; E-Mail: joachim. grzega@ku.de; ORCID: https://orcid.org/0000-0001-6562-5456. 
von Regierungserklärungen (s. Stüwe), Gesprächsprotokollen, Vertragsformulierungen (s. URL1) und Fernsehansprachen (s. URL2) exemplarisch illustriert werden. Wichtige Wörter werden unterstrichen.

\section{OSTBEZOGENE RHETORIK DER KANZLER VOR BRANDT}

Die gegenüber dem sowjetischen Einflussbereich praktizierte westdeutsche Politik ab 1949 wird gern als „Politik der Stärke“ und „Abschreckung“ etikettiert. Betrachten wir die Regierungserklärungen der deutschen Bundeskanzler nach dem Zweiten Weltkrieg, dann fällt auf, dass die Kanzler in ihren wegweisenden Regierungserklärungen stets die DDR-Frage ansprachen. Durch die Bezeichnung „Ostzone“ betrachtet Konrad Adenauer (CDU) in seiner Regierungserklärung von 1949 die DDR klar als Teil seines Landes. Zwar gäben Stalin 1952 und Chruschtschow 1953 ihr Einverständnis zur Wiedervereinigung, wenn ein Gesamtdeutschland neutral wäre. Adenauer geht darauf jedoch nicht ein und verstärkt Konfrontationen durch den Ausdruck „sowjetisch besetzte Zone“ in seiner Regierungserklärung von 1953. Ab 1955 propagiert Chruschtschow „friedliche Koexistenz“, wobei er sich in Bezug auf eine Wiedervereinigung nach Annäherung der beiden Deutschlands positiv eingestellt zeigt. In der BRD hingegen wird 1955 unter Adenauer die Hallstein-Doktrin formuliert: laut dieser werde jede Aufnahme diplomatischer Beziehungen zur DDR durch andere Staaten als ,unfreundlicher Akt" gegenüber der BRD erachtet. Zudem wird die BRD militärisch gestärkt durch Aufnahme in die NATO. Ferner verhält sich Adenauer während seines Besuchs in Moskau ungeschickt bis konfrontativ, wie Akten verraten (s. URL3). So heißt es in einem Dolmetscher-Protokoll:

Bemerkungen des Herrn Bundeskanzlers auf dem Flugplatz bei der Ankunft in Moskau am 8.9.55.: Nach Beendigung der Empfangszeremonie wurde der Herr Bundeskanzler von den anwesenden deutschen Journalisten angerufen und gebeten, mit Ministerpräsident Bulganin sich den Fotografen zu stellen. Er kam dieser Aufforderung nach und sagte dabei zu Herrn Bulganin „Das sind die eigentlichen Diktatoren“.

Dies suggeriert freilich, dass auch Bulganin eine Art Diktator sei. Gemäß einem weiteren Dolmetscher-Protokoll attackiert Adenauer Bulganin und Chruschtschow mit Blick auf „,normale Beziehungen“, die die Sowjetunion bezweckt: 
Der Gedanke ist unerträglich, daß mehr als zehn Jahre nach Beendigung der Feindseligkeiten Menschen, die auf die eine oder die andere Weise in den Strudel der kriegerischen Ereignisse gezogen worden sind, ihren Familien, ihrer Heimat, ihrer normalen friedlichen Arbeit ferngehalten werden. Sie dürfen nichts Provozierendes darin finden, wenn ich sage: Es ist nicht denkbar, normale Beziehungen zwischen unseren Staaten herzustellen, solange diese Frage ungelöst bleibt.

\section{Bulganin entgegnet:}

Unserer Meinung nach handelt es sich hier um ein bestimmtes Missverständnis. In der SU gibt es keine Kriegsgefangenen. Alle deutschen Kriegsgefangenen sind befreit und in ihre Heimat zurückgebracht. In der SU befinden sich nur die deutschen Kriegsverbrecher aus der ehemaligen Hitler-Armee.

Obwohl es festes und bekanntes politisches Schlagwort ist, glaubt Adenauer außerdem leugnen zu können, dass es das Konzept der „Politik der Stärke" gebe:

Sie haben dann davon gesprochen, daß in Deutschland von der Position der Stärke geredet werde. Ich glaube, daß da ein sehr großes Mißverständnis vorliegt. Kein Mensch in Deutschland, auch der Verrückteste nicht, bildet sich etwa ein, mit der Sowjetunion aus der Position der Stärke heraus verhandeln zu können.

Ferner ergänzt er, was wie eine Art Aufrechnung oder Abrechnung wirkt:

Es ist wahr: Truppen sind in Rußland eingefallen. Es ist wahr: es ist viel Schlechtes geschehen. Es ist aber auch wahr, daß die russischen Armeen dann - in der Gegenwehr, das gebe ich ohne weiteres zu -, in Deutschland eingedrungen sind und daß dann auch in Deutschland viele entsetzliche Dinge im Krieg vorgekommen sind. Ich meine, [...] daß wir dann nicht zu tief in die Vergangenheit hineinsehen sollten, weil wir dann nur Hindernisse vor uns aufbauen.

Hierauf erklärt Chruschtschow, zunächst erbost, später beschwichtigend,

daß die sowjetischen Truppen, als sie die Grenzen der Sowjetunion überschritten haben und weiter in Ihr Land eindrangen, auch Verbrechen verübt haben. Ich lehne das kategorisch ab, da es nicht der Fall war, und keine Beweise kann uns die deutsche Seite dafür vorbringen. Die sowjetischen Truppen haben die anderen aus ihrem Lande verjagt und sie weiter verfolgt, weil sie sich nicht ergeben haben. [....] Und deshalb haben die sowjetischen Soldaten ihre heilige Pflicht vor der Heimat erfüllt, indem sie den Krieg fortsetzten und ihr Leben dabei opferten. [....] Wir haben nur das eine Ziel vor uns: die Sicherung des Friedens in der ganzen 
Welt. Wir suchen keine Freundschaft mit Ihnen dazu, um gegen andere Staaten [...] vorzugehen. [....]. Wir wollen normale Beziehungen zu Ihnen herstellen und Freundschaft mit Ihnen stiften. Eine ebensolche Freundschaft wünschen wir auch zwischen unserem Staat und Großbritannien, Frankreich und den USA und allen anderen Ländern herbeizuführen. Ich möchte damit schließen und um Entschuldigung bitten, wenn ich irgendwelche schroffen Worte gebraucht habe, die ich nicht gebraucht haben wollte.

In der Regierungserklärung von 1957 nennt Adenauer die DDR weiterhin „sowjetisch besetzte Zone“. Im August 1961 folgt der Bau der Berliner Mauer. Eine rhetorische Entschärfung erfolgt nicht. Adenauer bezeichnet die DDR in der Regierungserklärung 1961 als „sowjetische Besatzungszone“, sein Nachfolger Ludwig Erhard (CDU) sagt 1963 in seiner Regierungserklärung „sowjetisch besetzte Zone“.

In dieser konfrontativen Stimmung wird Willy Brandt (SPD) 1966 erstmalig Mitglied einer Regierung, und zwar als Außenminister in einer Großen Koalition aus CDU und SPD. Für eine Aussöhnung mit dem Osten hatte er mit seinem Weggefährten Egon Bahr 1963 den Begriff „Wandel durch Annäherung“ geprägt. In der Regierungserklärung von Kurt Georg Kiesinger (CDU) wird die DDR erstmals „der andere Teil Deutschlands“ genannt; es wird verkündet, dass man „den Unruheherd der deutschen Teilung ... durch friedliche Verständigung beseitigen und unserem Volk seinen Frieden mit sich und mit der Welt wiedergeben“"will. Den Worten folgen jedoch keine für die Ostseite zufrieden stellenden Taten. Als Reaktion wird 1967 die Ulbricht-Doktrin formuliert, nach der die Mitglieder des Warschauer Paktes ihre Beziehungen zur BRD nicht normalisieren dürfen, solange diese nicht „die bestehenden Grenzen und die Existenz zweier deutscher Staaten“ anerkennt. Ergänzt wird dies 1968 um die Breschnew-Doktrin, nach der die Sowjetunion für sich das Recht in Anspruch nehme, einzugreifen, wenn sie in einem der Staaten des Warschauer Pakts den Sozialismus bedroht sähe. Im Ostblock gelten die Westdeutschen als Leute, denen man nicht trauen kann, die im Grunde revisionistisch nur wieder die alten Machtbereiche herstellen wollen (s. etwa Tomala 283, Stokłosa 247f.). Kiesinger und Brandt werden als Gegenpole und die Außenpolitik als sprunghaft wahrgenommen (s. Fischer, „Willy Brandt” 55). 


\section{OSTBEZOGENE RHETORIK BRANDTS}

1969 wird Brandt Kanzler einer SPD/FDP-Koalition. Nun hat er Gelegenheit, seine Vorstellung von einem friedlichen Europa umzusetzen. Er muss dies nicht nur gegenüber Regierungen, Bevölkerung und Medien im Osten vermitteln, sondern auch gegenüber Regierungspartei(en), Opposition, Bevölkerung und Medien im Westen, insbesondere Hardlinern und Ängstlichen. Das Schlagwort „Politik der kleinen Schritte“ wird wichtig. „Wandel durch Annäherung" soll nun realisiert werden. Dazu bricht Brandt die bisherigen rhetorischen Grenzen auf, wofür Bahr oft die Vorarbeit leistet (s.a. Bahr). Schon 1960 scheint er sich bewusst, dass es eine neue Sprachregelung brauche (Schmidt 91f.), wozu seine vielfältigen Kenntnisse europäischer Sprachen beigetragen haben mögen. Dies spiegelt sich nun in der Regierungserklärung, die zu großem Unmut unter den konservativen Abgeordneten führt:

20 Jahre nach Gründung der Bundesrepublik Deutschland und der DDR müssen wir ein weiteres Auseinanderleben der deutschen Nation verhindern, also versuchen, über ein gere-geltes Nebeneinander zu einem Miteinander zu kommen. [....] Die Bundesregierung [...] bietet dem Ministerrat der DDR erneut Verhandlungen beiderseits ohne Diskriminierung auf der Ebene der Regierungen an, die zu vertraglich vereinbarter Zusammenarbeit führen sollen. Eine völkerrechtliche Anerkennung der DDR durch die Bundesregierung kann nicht in Betracht kommen. Auch wenn zwei Staaten in Deutschland existieren, sind sie doch für einander nicht Ausland; ihre Beziehungen zueinander können nur von besonderer Art sein. (Beifall bei den Regierungsparteien. - Unruhe bei der CDU/CSU.) Anknüpfend an die Politik ihrer Vorgängerin erklärt die Bundesregierung, daß die Bereitschaft zu verbindlichen Abkommen über den gegenseitigen Verzicht auf Anwendung oder Androhung von Gewalt auch gegenüber der DDR gilt. [....] Wir wollen ein Volk der guten Nachbarn sein und werden, im Inneren und nach außen. (Anhaltender lebhafter Beifall bei den Regierungsparteien. - Abg. Dr. Barzel: Das ist ein starkes Stück, Herr Bundeskanzler! Ein starkes Stück! Unglaublich! Unerhört!)

Zum ersten Mal verwendet ein Bundeskanzler in einer Regierungserklärung den Namen $D D R$. Ferner erkennt er die DDR als Staat an. Gleichzeitig signalisiert er für den Westen, dass damit keine völkerrechtliche Anerkennung verbunden sei und dass es eine Sonder-Beziehung geben müsse. Später oft zitiert wird das Wort „Wir wollen ein Volk der guten Nachbarn sein“, das CDU-Oppositionsführer Barzel zu erbosten Reaktionen reizt. 
Ebenso neu ist, dass ein Bundeskanzler verlautet, dass es Angebote aus der Sowjetunion und Polen gebe, und dass man auf diese eingehen wolle.

Sie [= die neue Bundesregierung] wird demnächst das sowjetische Aide-mémoire zum Thema Gewaltverzicht beantworten und einen Termin für die von der Sowjetunion angeregten Verhandlungen in Moskau vorschlagen. [...]. Sie wird der Regierung der Volksrepublik Polen einen Vorschlag zur Aufnahme von Gesprächen zugehen lassen, mit dem sie die Ausführungen Wladislaw Gomulkas vom 17. Mai dieses Jahres beantwortet.

Manche im Ostblock hatten sich wohl noch mehr erwartet (s. Falin 63). Außenminister Gomułka nimmt bei Brandt jedoch grundsätzlich positiv wahr, dass er als Initiator von Gesprächen genannt wird (Miszczak 286).

Schließlich sagt Brandt als Signal an den Osten Folgendes zu:

Sie [= die neue Bundesregierung] wird den Vertrag über die Nichtverbreitung von Atomwaffen unterzeichnen, sobald - entsprechend den Beschlüssen der letzten Bundesregierung - die noch ausstehenden Klärungen herbeigeführt sind. (Beifall bei den Regierungsparteien. - Lachen bei der CDU/CSU.)

Der Nebensatz bedeutet dem Westen, dass dies nur im übergreifenden Einverständnis sein werde. Brandt sucht also beide Seiten rhetorisch zu befrieden.

Der Atomwaffensperrvertrag wird von Brandt bereits einen Monat später unterschrieben. Er setzt sein Wort also zeitnah in die Tat um, was im Osten positiv auffällt (s. Falin 66).

Die ersten Begegnungen mit DDR-Ministerratsvorsitzenden Stoph verlaufen wenig erfolgreich, obschon Brandt vermutlich klug zurückhaltend reagiert, als die anwesende DDR-Bevölkerung vor dem Erfurter Rathaus begeistert ruft: „Willy Brandt ans Fenster!“. Damit vermeidet er, dass sich die DDR-Führung brüskiert fühlt.

Nach dem Gegenbesuch entsteht jedoch das Wort der „Denkpause“, die man sich nun gebe. Brandt erkennt, dass der Weg nur über Moskau gehen wird (s.a. Bahr). Ferner versteht er, dass es Wörter gibt, die für den Osten zentral sind. So gelingt es, dass 1970 der Moskauer Vertrag zwischen der BRD und der Sowjetunion unterzeichnet wird. Als Unterzeichnungsdatum bevorzugt Brandt den 12. statt des 13. Augusts, des Tags des Mauerbaus; er wünscht keinen Zusammenfall zweier gegensätzlicher symbolträchtiger Ereignisse (Falin 112; Fischer, „Einleitung“ 53). Im Dezember 1970 reist 
Brandt zur Unterfertigung des Warschauer Vertrags. In beiden Verträgen geht es um Grenzanerkennung, Gewaltverzicht und bessere Beziehungen. Wichtige symbolhafte Wörter werden von Bahr und Brandt bewusst aufgenommen oder vermieden. So werden Formulierungen wie „Oder-NeißeLinie [poln.: Granica na Odrze i Nysie Łużyckiej]“" ,achten/nicht antasten/ unverletzlich“ als wichtig erkannt (s.a. Miszczak 55; Stokłosa 230). Brandt weiß, dass dies vor allem für deutsche Heimatvertriebene schmerzlich ist. Doch in einer Umfrage des polnischen Nachrichtenmediums Polityka waren $80,6 \%$ der Befragten für eine Aufnahme von Gesprächen mit Deutschland nur, wenn die Oder-Neiße-Linie anerkannt würde (Miszczak 293). Auch für Moskau ist es belangreich (s. Zündorf 38f.). Ferner lässt Brandt das aus sowjetischem Munde stammende Wort der „Normalisierung“ einbauen. Eine mündliche Äußerung Brandts, die „Normalisierung“ sei der wesentliche Punkt des Vertrages, wird von Ministerpräsident Cyrankiewicz zustimmend zur Kenntnis genommen (Miszczak 65). Vermieden wird hingegen mit Blick auf die deutsche und übrige westliche Bevölkerung der Ausdruck ,völkerrechtlich [anerkennen]"; dem Verzicht auf dieses Wort kann die Sowjetunion zustimmen (Zündorf 44).

Unmittelbar nach Unterzeichnung des Moskauer Vertrags wendet sich Brandt in einer Fernsehansprache an die deutsche Bevölkerung, mit wohlbedachten Worten, die bald für die eine Seite, bald für die andere Seite positiv beladen sind. Er verkündigt das

Bestreben, die Normalisierung der Lage in Europa und die Entwicklung friedlicher Beziehungen zwischen allen europäischen Staaten zu fördern [....] in Fragen der Gewährleistung der europäischen und der internationalen Sicherheit von den Zielen und Grundsätzen, die in der Charta der Vereinten Nationen niedergelegt sind, leiten lassen

[....] Streitfragen ausschließlich mit friedlichen Mitteln lösen [....] die heutigen Grenzen uneingeschränkt zu achten [...] [BRD \& UdSSR] betrachten heute und künftig die Grenzen aller Staaten in Europa als unverletzlich, wie sie am Tage der Unterzeichnung dieses Vertrages verlaufen, einschließlich der Oder-Neiße-Linie, die die Westgrenze der Volksrepublik Polen bildet, und der Grenze zwischen der Bundesrepublik Deutschland und der Deutschen Demokratischen Republik. [....] Dieser Vertrag [....] berührt nicht die von ihnen früher abgeschlossenen zweiseitigen und mehrseitigen Verträge und Vereinbarungen [und] bedarf der Ratifikation [...].

Als Gomułka einmal fragt „Wozu brauchen wir die Amerikaner?“, betont Brandt gemäß Gesprächsprotokoll die Wichtigkeit von Bindungen zur Vermeidung von Nationalismus (Tomala 303). Daher bedankt sich Brandt bei 
US-Präsident Richard Nixon auch für die Unterstützung; allerdings stellt Nixon klar, dass es keine Unterstützung war, sondern nur ein Nicht-Opponieren (Stokłosa 207). So zeigt sich wie auch im weiteren Verlauf, dass die Wortwahl zentral ist. Einem Protokoll von einem Gespräch mit KPdSU-Generalsekretär Breschnew (Pautsch 1456) entnimmt man, dass Brandt Wörter bewusst mit Blick auf den jeweiligen Adressaten harmonisch variieren will und dies auch auf der Meta-Ebene tut:

Breschnew: [....] Scheel sagte [in einem Interview]: „Wir haben offiziell erklärt, daß dieser Vertrag nicht in Kraft gesetzt werden kann, bevor nicht eine positive Lösung in der Berlin-Frage erreicht ist." Was bedeutet das? [....] Sie kennen unsere Position in bezug auf Westberlin. Ich möchte nicht ausschließen, daß bei gutem Willen aller Vier Mächte man Kompromisse finden kann, die annehmbar für alle sind und bestimmte Hoffnungen geben können. Wenn Herr Scheel dies mit Ihrer Zustimmung etwa aus Propagandagründen gesagt hat, um den Ratifizierungsprozeß im Bundestag zu erleichtern, so könnte ich das verstehen, aber wenn diese Worte von Scheel den wirklichen Standpunkt der Bundesregierung wiedergeben, wie sollen wir das dann verstehen? Bedeutet das, daß die Bundesregierung Fragen unseres Vertrages an die USA abgibt? D. h. doch, die Bundesregierung gibt den USA ein Vetorecht. [....]

Was den Hinweis auf das Interview von Außenminister Scheel anbelangt, verwies der Herr Bundeskanzler auf das, was am Rande der Verhandlungen erörtert wurde und was der Außenminister gegenüber Gromyko erklärt hat. Wir sehen die Regelung mit der SU, der VRP, der ČSSR und der DDR als Ganzes, aber die angestrebte Regelung mit der DDR bleibt eine halbe Sache, wenn es zu keiner Beruhigung im Herzen Europas komme. [....] Es gibt kein juristisches Junktim, sondern die bekannte Erklärung unseres Außenministers. Aber die politische und psychologische Lage in der Bundesrepublik ist so, daß wir eine breitere Zustimmung für diesen Vertrag erhoffen, wenn es Fortschritte in bezug auf Berlin gibt. [....] sieht sich ab dem heutigen Tag politisch an den Vertrag gebunden und wird nach diesem Vertrag handeln. Die Bundesregierung räumt auch den besten Freunden kein Vetorecht ein.

\section{$[\ldots$.}

[Bundeskanzler:] Die Bundesregierung strebe trotz aller Besonderheiten zu einer vertraglichen Regelung mit der DDR, die ebenso verbindlich sei wie Verträge mit sonstigen Staaten. [....]

Breschnew. Ich verstehe, daß diese vertragliche Regelung das beinhalten wird, wonach die DDR strebt, nämlich rechtliche Anerkennung.

Bundeskanzler: „Anerkennung“ sei ein umstrittener Begriff. Bundesregierung will Kategorien wie territoriale Integrität und Unverletzlichkeit der Grenzen und an- 
dere Kategorien des zwischenstaatlichen Rechts zu Elementen eines Vertrages machen.

$[\ldots$.

Breschnew: [....] Abwarten würde die Lösung anderer Fragen, u. a. auch der Westberlin-Frage, verzögern, die ja keine juristische Bindung an diesen Vertrag hat. Ich bitte, das zu bedenken. Andererseits könnte eine baldige Ratifizierung dazu beitragen, daß keine Verwirrung entsteht, da $\beta$ andere Fragen eine positive Lösung finden. Der Herr Bundeskanzler betonte, [....] [die] Sache des Vertrages mit der Sowjetunion sei [...] nicht nur eine Sache seiner Partei oder der Partei von Herrn Scheel, sondern eine Sache des Volkes, insbesondere der jungen Generation. Der Herr Bundeskanzler verwies auf die große Unterstützung, die eine Meinungsumfrage ergeben habe. Er wolle wiederholen, das Mitgetragenwerden durch die Bevölkerung sei leichter, wenn beide Seiten in schwierigen Punkten, auch Berlin, Lösungen erzielten.

In einer Fernsehansprache zur Ratifizierung des Moskauer Vertrags sagt Brandt an Deutschland:

mit diesem Vertrag geht nichts verloren, was nicht längst verspielt worden war. [....] Dieser Vertrag beeinträchtigt in keiner Weise die feste Verankerung der Bundesrepublik und ihrer freien Gesellschaft im Bündnis des Westens. Die zuverlässige Partnerschaft mit Amerika bleibt ebenso gewahrt wie die Aussöhnung mit Frankreich. Es bleibt auch bei dem beharrlichen Willen, immer mehr Staaten Europas mit dem Ziel einer politischen Gemeinschaft immer enger aneinander zu binden. Der Vertrag gefährdet nichts und niemanden. Er soll mithelfen, den Weg nach vorn zu öffnen. Wenn er dies tut, dann wird er dem Frieden, Europa und uns allen nützen.

Im Umfeld des Warschauer Vertrags kommt es zu dem später so symbolhaften stummen Kniefall Brandts im Warschauer Ghetto - wortlose Friedensrhetorik. Nach Vertragsunterzeichnung hält Brandt eine Fernsehansprache, worin er erneut betont:

Was ich im August Ihnen aus Moskau gesagt habe, liebe Mitbürgerinnen und Mitbürger, gilt auch für den Vertrag mit Polen: Er gibt nichts preis, was nicht längst verspielt worden ist. Verspielt nicht von uns, die wir in der Bundesrepublik Deutschland politische Verantwortung tragen und getragen haben. Sondern verspielt von einem verbrecherischen Regime, vom Nationalsozialismus. [....] Wir dürfen nicht vergessen, dass dem polnischen Volk nach 1939 das Schlimmste zugefügt wurde, was es in seiner Geschichte hat durchmachen müssen. Dieses Unrecht ist nicht ohne Folgen geblieben. Großes Leid traf auch unser Volk, vor allem unsere ostdeutschen Landsleute. 
[....] Wir müssen unseren Blick in die Zukunft richten und die Moral als politische Kraft erkennen. [....]

Der Vertrag zwischen Polen und uns [....] ersetzt keinen formellen Friedensvertrag. Er berührt nicht die Rechte und Verantwortlichkeiten der Vier Mächte für Deutschland als Ganzes. Er setzt frühere vertragliche Verpflichtungen weder der einen noch der anderen Seite außer Kraft.

Auch für das informelle Gespräch, den Small-Talk, sucht Brandt offenbar zu lernen. So war er bei seinem Besuch in Moskau im August 1970 angeblich überrascht von der Frage des russischen Premiers Kossygin „Und wie war die Ernte?" und missinterpretierte sie wohl als jederzeit im Ostblock angebrachte Small-Talk-Frage. Allerdings ist diese nur nach der Erntezeit angebracht. Jedenfalls stellte er diese Frage auch im Dezember, also Winter, 1970 bei seinem Treffen mit dem polnischen Ministerpräsidenten Cyrankiewicz. Dieser war zunächst verdutzt über diese saisonal seltsame Frage, konnte aber im Nachhinein vom Dolmetscher aufgeklärt werden, der auch dem Treffen in Moskau beigewohnt und damals Brandts Verwunderung über die Frage beobachtet hatte (Tomala 302). Ungeachtet dieser misslungenen Kommunikationsepisode Brandts heben Falin (124) und Stokłosa (249) positiv die guten Kontakte hervor, die Brandt über informelle Kommunikation herstellen konnte. Gegenüber dem Oppositionsführer Carstens soll er gesagt haben, man müsse im Kontakt mit Vertretern des anderen Lagers „leise wirken“" (s. Schmidt 86).

Bei der Verfassung einer Übersetzung zum Viermächteabkommen über Berlin im darauffolgenden Jahr, 1971, beginnen die Vertreter der DDR plötzlich Streitigkeiten zur deutschen Übersetzung von engl. Ties / frz. liens / russ. связи, was Brandt zunächst erbost, weil er Sabotage annimmt (Falin 176). Schließlich gibt es zwei inoffizielle Übersetzungen, nämlich Bindungen in der westdeutschen und Verbindungen in der ostdeutschen Übersetzung.

Im Dezember 1971 kann Brandt den Friedensnobelpreis entgegennehmen. Seine Festrede versieht er wiederum mit einer Reihe von symbolträchtigen Wendungen für beide Seiten, bis hin zu Gleichgewicht, welches das britische geopolitische Ziel der „balance of powers“ ins Bewusstsein ruft:

Ich möchte [...] betonen, dass meiner Überzeugung nach die gesamteuropäische Sicherheit und Zusammenarbeit nicht beeinträchtigt wird, wenn die westeuropäische Einigung weiter voranschreitet. Westeuropa mit Einschluss Großbritanniens, also die sich erweiternde Gemeinschaft, formiert sich nicht als Block gegen den Osten, sondern 
kann - auch durch die Stärkung ihrer sozialen Komponente - zu einem besonders wichtigen Bauelement einer ausgewogenen europäischen Sicherheit werden. [...]. Weiter will ich sagen: Europa und Amerika sind nicht zu trennen. Sie brauchen einander als selbstbewusste, gleichberechtigte Partner. [...]. Unsere gesamteuropäische Politik kann über die jahrhundertealten Identitäten von Nationen und Staaten nicht hinweggehen. Wir müssen vielmehr ein Gleichgewicht zwischen den Staaten und Staatengruppen schaffen und wahren, in dem die Identität und die Sicherheit eines jeden von ihnen geborgen sein kann. Ein solches Gleichgewicht muss aber mehr sein als nur ein ausgewogenes System militärischer Machtmittel. [....]. Europäische Friedenspolitik lebt aus dem Geist der Geschichte. Dies klammert die dunkelsten Jahre nicht aus, sondern bezieht sie ausdrücklich ein. [....] Ein Europa des Friedens braucht die Bereitschaft zum Hinhören auf die Argumente des anderen, denn das Ringen der Überzeugungen und Interessen wird weitergehen. Europa braucht Toleranz. Nicht moralische Gleichgültigkeit, sondern Gedankenfreiheit. [....] Friede ist mehr als Abwesenheit von

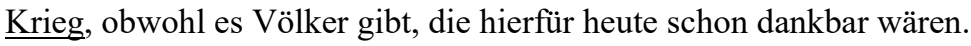

Es folgen die Abkommen mit der DDR. Am längsten dauern die Verhandlungen mit der Tschechoslowakei. Der Prager Vertrag kommt auch hier nach einer „Denkpause“ erst 1973 zu Stande. Im Wesentlichen geht es um das Münchner Abkommen von 1938, das im Vertrag als „nichtig“ erklärt wird. Bewusst wird im Konsens nur das Wort „nichtig“ gesetzt, um späteren juristischen Diskursen offen zu lassen, ob dieses „ex tunc“ 'von Anfang an' oder „ex nunc“ 'ab jetzt' zu verstehen ist (s. Zürndorf 101).

Der Furcht vieler Franzosen vor einem übermächtigen Deutschland und einem starken deutsch-sowjetischen Bündnis begegnet Brandt, indem er wiederholt die Westbindung Deutschlands hervorhebt, den Begriff „entente élémentaire“ 'Elementares Verständnis' schöpft (in Anlehnung an die „entente cordiale“" 'herzliches Verständnis' zwischen Frankreich und Großbritannien) und dieses in seine Regierungserklärung von 1973 einbaut.

\section{NACHWEIS DES ERFOLGS}

Der langfristige Friedenseffekt der Politik von Brandt und Bahr kann hier nicht näher beleuchtet werden. Trotz der Komplexität durch die inneren Erfolge (Müller) soll jedoch auf das eingegangen werden, was als ziemlich direkte Reaktion auf Brandts Rhetorik verstanden werden kann. So sagt etwa zwei Tage nach Brandts erster Regierungserklärung DDR-Staatsratsvorsitzender Ulbricht: „Die Regierung Willy Brandt stellt einen Fortschritt dar“ (Stokłosa 231). Später lässt Ministerpräsident Cyrankiewicz Brandt wissen, 
dass seine Aussagen über die deutsch-polnischen Beziehungen mit Zufriedenheit aufgenommen wurden (Stokłosa 230). Die öffentliche Meinung über Brandt ist so hoch, dass US-Präsident Nixon und US-Außenminister Kissinger sich gezwungen sehen, trotz ihres Missfallens Brandt zum Vertragswerk zu gratulieren (Stokłosa 254). Das Time Magazine kürt in seiner Ausgabe vom 04.01.71 Brandt zum „Man of the Year“. Ebenfalls 1971 wird ihm der Friedensnobelpreis verliehen. Innerhalb von Deutschland lässt sich gemäß einer Allensbach-Umfrage beobachten, dass sich im November 1968 noch 54\% von der UdSSR bedroht fühlen, im April 1971 nur noch 28\%; gemäß einer Emnid-Umfrage unterstützen 59\% Brandts Ostpolitik; Allensbach-Umfragen im März, April und Mai 1972 zeigen, dass eine klare Mehrheit die Ratifizierung der Ostverträge befürwortet: 57\%, 57\% und 61\% (s. Stokłosa 298). Zur Befriedung der Opposition, die Brandts Politik als deutschen Interessen entgegenstehend bezeichnete (Stokłosa 287; Meissner), einigt man sich auf eine gemeinsame Entschließung aller Bundestagsfraktionen, die im Kern besagt, dass die Verträge nur als Übergangsregelung zu verstehen seien und die Friedensverträge noch ausstünden. Mag dies im Ostblock auch irritieren, so scheint dies wiederum wichtig, um der Politik der aktiven Friedenssicherung im eigenen Land Akzeptanz zu verschaffen. Auch die Ängste der Franzosen schwinden, indem etwa Brandts Beteuerungen von Leuten wie Joseph Rovan und Alfred Grosser verbreitet werden (s. Petter 100f.).

\section{LITERATUR}

Bahr, Egon. „Das musst du erzählen. “Erinnerungen an Willy Brandt. Ullstein, 2013.

Falin, Valentin. Politische Erinnerungen. Droemer Knaur, 1995.

Fischer, Frank. „Willy Brandt und die Deutschland- und Außenpolitik 1966-1982“. Stand und Perspektiven der Willy-Brandt-Forschung: Werkstattgespräch zur Berliner Ausgabe, hrsg. von Wolfgang Schmid, Bundeskanzler-Willy-Brandt-Stiftung, 2003, S. 53-62.

Fischer, Frank. „Einleitung“. Willy Brandt. Berliner Ausgabe, hrsg. von Helga Grebing, Gregor Schöllgen und Heinrich August Winkler, vol. 6, Dietz, 2005, S. 15-92.

Grzega, Joachim. Wort-Waffen abschaffen! Beobachtungen zu Europas gewaltvoller Wortwahl und Ideen für eine friedensstiftende Sprache. epubli, 2019.

Meissner, Lucjan. „Willy Brandts Versöhnungspolitik gegenüber Polen 1970-1972: Ein Erinnerungsbeitrag“. Zeitschrift des Verbandes polnischer Germanisten 4/4, 2015, S. 309-318.

Miszczak, Krzysztof. Deklarationen und Realitäten. Die Beziehung zwischen der Bundesrepublik Deutschland und der (Volks-) Republik Polen von der Unterzeichnung des Warschauer Vertrages bis zum Abkommen über gute Nachbarschaft und freundschaftliche Zusammenarbeit (1970-91). tuduv, 1993. 
Müller, Albrecht. Brandt aktuell. Treibjagd auf einen Hoffnungsträger. Westend, 2013.

Pautsch, Ilse Dorothee et al. Akten zur Auswärtigen Politik der Bundesrepublik Deutschland. 1970. vol. 1. Oldenbourg, 2001.

Petter, Dirk. Auf dem Weg zur Normalität: Konflikt und Verständigung in den deutsch-französischen Beziehungen der 1970er Jahre. De Gruyter, 2014.

Schmidt, Wolfgang (Hrsg.). Stand und Perspektiven der Willy-Brandt-Forschung. Werkstattgespräch zur Berliner Ausgabe. Bundeskanzler-Willy-Brandt-Stiftung, 2003.

Stokłosa, Katarzyna. Polen und die deutsche Ostpolitik 1945-1990. Vandenhoeck \& Ruprecht, 2011.

Stüwe, Klaus, Herausgegeber. Die Großen Regierungserklärungen der deutschen Bundeskanzler von Adenauer bis Schröder. Leske und Budrich, 2002.

Tomala, Mieczysław. Deutschland - von Polen gesehen. Zu den deutsch-polnischen Beziehungen 1945-1990. Schüren, 2000.

URL1 = Kai Riedel. document Archiv. BRD, http://www.documentarchiv.de/brd.html. Abruf am 18.04.2020.

URL2 = Willy-Brandt-Stiftung. Reden, https://willy-brandt.de/willy-brandt/reden-zitate-und-stim$\mathrm{men} / \mathrm{reden} /$. Abruf am 18.04.2020.

URL3 = Konrad-Adenauer-Stiftung. Moskaureise, https://www.konrad-adenauer.de/quellen/ moskau reise. Abruf am 18.04.2020.

Zündorf, Benno. Die Ostverträge. Beck, 1979.

\section{EUROLINGUISTYCZNE SPOJRZENIE NA WILLY'EGO BRANDTA - PROMOWANIE POKOJU PRZEZ PRZEZWYCIĘŻANIE RETORYCZNYCH GRANIC}

$$
\text { Streszczenie }
$$

Lata 1969-1971 są ważne w kontekście zakończenia „zimnej wojny”: wyznaczają początek i pierwsze sukcesy nowej polityki zagranicznej Willy'ego Brandta. Na podstawie deklaracji rządowych, tekstów umów, protokołów rozmów i wystąpień telewizyjnych w artykule pokazano, że jedną z ,cegiełek” do sukcesu Brandta stanowi przezwyciężenie dotychczasowych granic retorycznych. Jego retoryka wyróżnia się: (1) szybkim wcielaniem słów w czyny, (2) świadomością specyficznych dla kultury słów-symboli, (3) tworzeniem nowych słów-symboli o pozytywnym wartościowaniu, (4) wariacją słów w zależności od adresata, (5) (dobrą) komunikacją z osobami sprawującymi władzę.

Słowa kluczowe: Willy Brandt; promowanie pokoju; granice retoryki; eurolingwistyka.

\section{EUROLINGUISTIC LOOK AT WILLY BRANDT: PROMOTING PEACE THROUGH OVERCOMING RHETORIC BORDERS}

\section{Su m mary}

1969 to 1971 are important years in the end of the Cold War: the beginning and initial successes of Willy Brandt's new foreign policy. By means of government declarations, texts of treaties, records of conversation and TV speeches, this article illustrates that one constituent of Brandt's 
success lay in his overcoming the traditional limits of rhetoric. His rhetorical style features: (1) the prompt implementation of words into action, (2) an awareness of culture-specific symbolic words, (3) the creation of new positive symbolic words, (4) addressee-specific word variation, and (5) (good) communication with political rulers (and an avoidance of their opponents).

Keywords: Willy Brandt; promoting peace; limits of rhetoric; eurolinguistics. 